\title{
LE CONTEXTE GÉOMORPHOLOGIQUE ET LES ACTIVITÉS SISMIQUE UNE APPLICATION : SÉISME DE 14NOVEMBRE 1981 SUD DE L'EGYPTE \\ Dr. M. Arfan
}

\begin{abstract} de la théorie de la tectonique des plaques.

\section{-La méthodologie}

Afin d'étudier, les risques séismiques, il est nécessaire de voir si les conditions géologiques, et d'occupation du sol au sud de l'Egypte correspondent à des conditions "favorables" à ce risque.

L'étude des risques naturels présente, actuellement, un intérêt particulier pour la compréhension des mécanismes déclenchant ces risques. Leur identification est devenue une nécessité dans les politiques d'aménagement du territoire. L'information géomorphologique s'avère utile à différents niveaux de décision pour rationaliser les actions à entreprendre en prévision des catastrophes naturelles.

L'approche cartographique, basée sur la méthode géomorphologique, constitue un document scientifique de décision nécessaire aux actions à entreprendre. Il existe plusieurs méthodes et techniques de représentation cartographique des formes de risques. Chacune de ces méthodes se fonde sur des principes fondamentaux adaptés au objectif de cette recherché.
\end{abstract}

Dans cette recherche l'étude des séismes présente deux aspects : d'une part manifestation de l'activité interne du globe, d'autre part outil de compréhension de la structure du globe. En plus le remplissage initial d'eau des réservoirs de ces barrages qui a déjà causé des tremblements de terre. Car l'eau augmente considérablement la pression sur les failles préexistantes dans la roche. C'est donc dans le cadre général de l'activité du globe dont la sismicité et le volcanisme sont les deux principaux aspects que l'on peut étudier le modèle actuel de l'activité du globe ou modèle géodynamique global, héritier

Mots clé : Séisme- barrages - Assouan - réservoirs- Egypte- lac Nasser.

\section{-Problématique de cette recherche}

Le sujet de cette recherché est parmi les nouveautés d'étude en Egypte, cela a nécessité la lecture de toutes les études qui ont été faites sur les risques naturels et ayant trait aux risques géomorphologiques. Enfin, on a trouvé des contraintes pour avoir les données lithologiques et sur les séismes. Généralement nous avons trouvé avec difficulté les documents nécessaires à l'écriture de cette recherché. D'autre part l'absence de moyens de transport sur le terrain présente un obstacle majeur pour cette étude.

\section{Origine des tremblements de terre à} Assouan ?

Le 14 Novembre 1981 à 11 heures du matin, heure locale, une violente secousse a ébranlé la région. Elle était d'une magnitude de 5,3 sur l'échelle de Richter, (fig.1). Ce tremblement de terre a été précédé par trois secousses datant du 11, 12 et du 13 novembre 1981 et suivi d'un grand nombre de répliques sismiques qui ont continué à se produire jusqu' en janvier 1984. Cette activité est censée avoir été déclenchée par le lac (Kebeasy, 1982), comme le tremblement de terre produit le 12 janvier 1982 à 3 heures $41 \mathrm{mn}$ du matin, heure locale, d'une magnitude 4,2 sur l'échelle Richter, et le même jour 5 minutes après un autre tremblement de terre d'une magnitude 4 sur l'échelle de Richter.

Le 20 Août 1982 à 15 heures $50 \mathrm{mn}$, heure locale, un tremblement de terre s'est produit de 4,7 sur l'échelle de Richter. Pendant le jour même, le séismographe a enregistré 116 séismes (Dessokey, 1985). Le 24 février 1983 à 2 heures $33 \mathrm{mn}$, un tremblement de terre s'est produit de 4,3 sur l'échelle de Richter. La station télémétrique de Kalabsha a enregistré, le 25 février 1983, plus de 56 tremblements de terre de 3,5 et 4 sur l'échelle Richter dans la région d'Assouan.

Lorsqu'un matériau rigide est soumis à des contraintes de cisaillement, il va d'abord se déformer de manière élastique, puis, lorsqu'il aura atteint sa limite d'élasticité, il va se rupturer, en dégageant de façon instantanée toute l'énergie qu'il a accumulé durant la déformation élastique. C'est ce qui se passe lorsque la lithosphère est soumise à des contraintes. Sous l'effet des contraintes causées le plus souvent par le mouvement des plaques tectoniques, la lithosphère accumule l'énergie. Lorsqu'en certains endroits, la limite d'élasticité est atteinte, il se produit une ou des ruptures qui se traduisent par des failles. L'énergie brusquement dégagée le long de ces failles cause des séismes (tremblements de terre).

Dans le but de bien surveiller l'activité sismique dans la région d'Assouan, neuf stations télémétriques ont été installées sur les bordures 
Nord du lac. Elles ont commencé à fonctionner à partir de juillet 1982 (fig.2) Des milliers de micro tremblements ont été enregistrés. Les différentes stations télémétriques ont montré que l'activité séismique est concentrée essentiellement en trois zones.

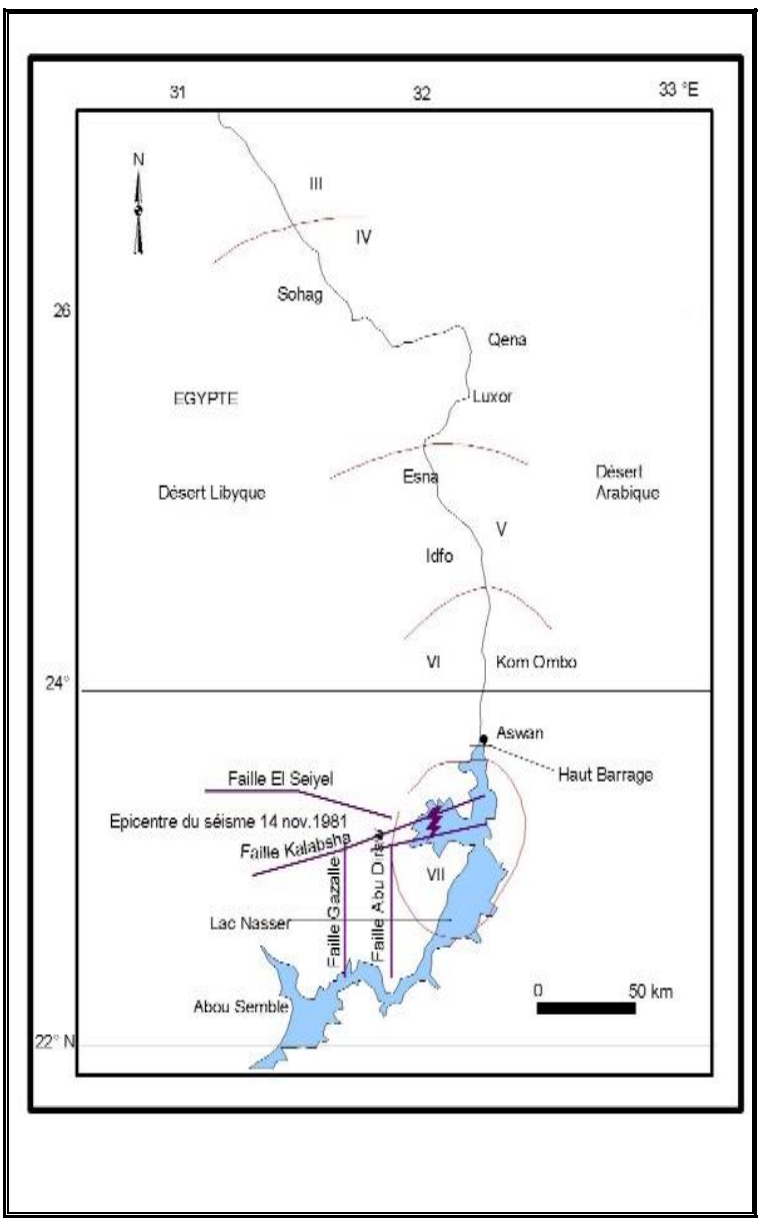

Figure 1. Répartition de l'intensité sismique du séisme d'Assouan du 14 novembre 1981 (D'après Kebeasy, 1982)

\section{2-1. Les zones d'activités sismiques}

Le premier secteur se prolonge Est/Ouest, sur environ 14 kilomètres, le long de la faille de Kalabsha. Les points de départ des séismes sont concentrés à deux intervalles de profondeur de 14 à 22 kilomètres et de 4 à 7 kilomètres (Woodward, 1985).

Le deuxième secteur se prolonge sur 8 kilomètres le long de l'autre segment de la faille de Kalabsha qui est plus près de l'ancien cours du Nil. Dans cette zone, l'activité est confinée aux profondeurs de 4 à 6 kilomètres. Concernant la distribution des points de départ des séismes dans ces deux zones, on observe une diminution progressive des profondeurs d'Ouest- Est.

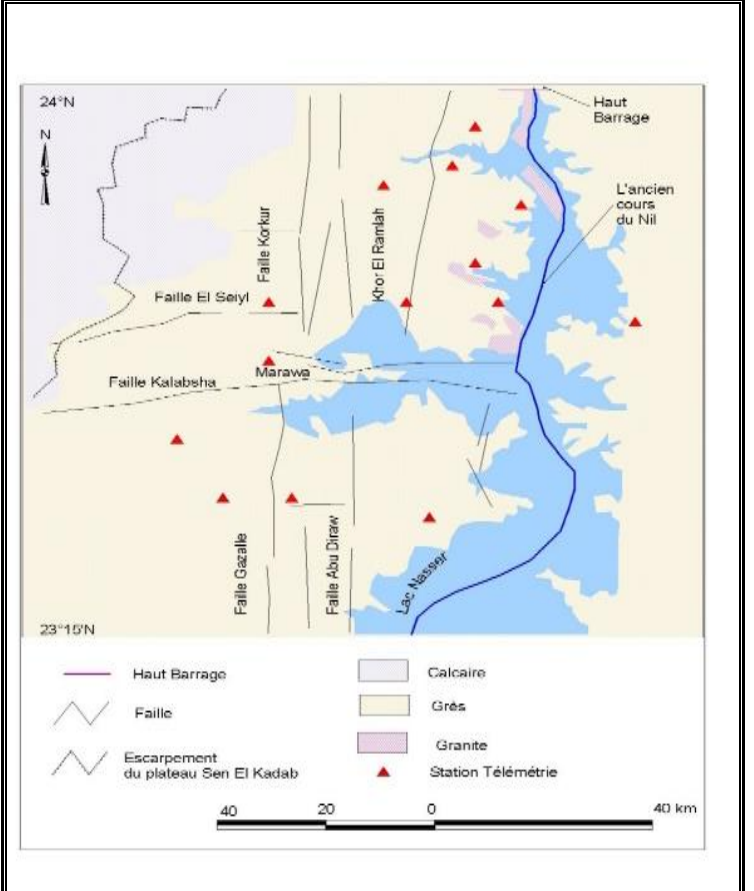

Figure 2. Localisation des réseaux télémétriques autour de la partie Nord du Lac Nasser (D'après, Dessokey, 1985)

Le troisième secteur est situé dans le secteur oued Korkur dans la partie Nord /Ouest du lac, environ 15 kilomètres au Sud du haut barrage d'Assouan. Ce secteur se caractérise par de faibles séismes.

Les résultats des mesures radios télémétriques font apparaître les remarques suivantes: Le premier et deuxième secteur se caractérise par une direction Ouest Sud-Ouest, Est Nord-Est. La distribution dans le temps indique une corrélation étroite de la séismicité avec les fluctuations saisonnières du niveau d'eau du lac. Le séisme du 14 novembre de 1981 semble être produit sur la faille de Kalabsha sous du Jbel Marawa (Kebeasy, 1987).

\section{2-2. Les facteurs provoquant la séismicité à Assouan}

Assouan appartient à une région qui situant à l'abri des activités sismiques. Elle se situe entre la formation granitique du désert arabique et la formation de calcaire et de sable consolidée du désert libyque. De plus, à ce potentiel séismo-tectonique, se couple un contexte géologique favorable à la propagation et à l'amplification des ondes séismiques (Hemdan, 1984). 
2-2-1. La tectonique et la séismicité de la région du Lac Nasser

Dans les roches dures et compactes, les séismes ont pour effet de créer des fissures, car ces roches deviennent cassables du fait de leur rigidité. La structure géologique fait varier l'intensité des séismes. Une forte intensité est souvent associée à des zones de roches meubles (sable et argile). A l'opposé, on note une faible intensité dans les zones de roches plus solides (grès).

Deux ensembles de systèmes de failles sont dominants dans le secteur : les failles nordsud sont très importantes et affectent la formation de Nubie. Le système est-ouest de faille est également constitué de failles normales et se prolonge sur plus de 300 kilomètres.

\section{2-2-2. Le contexte géologique de Kalabsha}

La région de Kalabsha se situe entre la latitude $23^{\circ} 20^{\prime}$ et $23^{\circ} 58^{\prime} \mathrm{N}$, et longitudes $32^{\circ}$ $00^{\prime}$ et $32^{\circ} 50^{\prime} \mathrm{E}$ (fig.2). La région de Kalabsha est relativement plane avec quelques escarpements qui se prolongent sur des dizaines de kilomètres. L'altitude du secteur est faible et se trouve entre 138 et $150 \mathrm{~m}$. La partie occidentale et la partie septentrionale sont généralement planes surtout dans la partie Nord.

Le socle affleure dans le Nord et le NordEst du plateau Sin El kadab. La topographie de ce secteur se compose de trois parties géomorphologiques distinctes :

1- Plaine de Nubie

2- Escarpement du plateau Sen El Kadab

3- Plateau de Sen El Kadab

La plaine de Nubie, avec environ du 20 $000 \mathrm{~km}^{2}$ s'étend du Nil au plateau de Sin El kadab. La plaine a peu de relief et l'altitude moyenne varie entre 138 et $174 \mathrm{~m}$, mais l'altitude du Jbel Marawa est d'environ 274 m dans du calcaire résistant (Issawi , 1978). Par ailleurs, la plaine est limitée Sud par du oued Kalabsha. Elle est couverte par l'eau du lac pendant les périodes d'inondations où le niveau d'eau du lac est à plus de $174 \mathrm{~m}$

Dans le secteur de l'Oued Kalabsha, il y a deux directions de failles, Est-Ouest et NordSud. Selon le séisme du 14 novembre 1981 et des secousses sismiques qui l'ont suivie, la faille active était la faille de Kalabsha. Cette faille s'étend d'Oued Kalabsha à l'escarpement du plateau Sin El Kadab (fig.3) en passant par le Jbel Marawa. Dans ce secteur, s'est développée une grande faille du côté oriental du Jbel
Marawa formée de plusieurs tracés parallèles, d'une direction Est -Ouest.

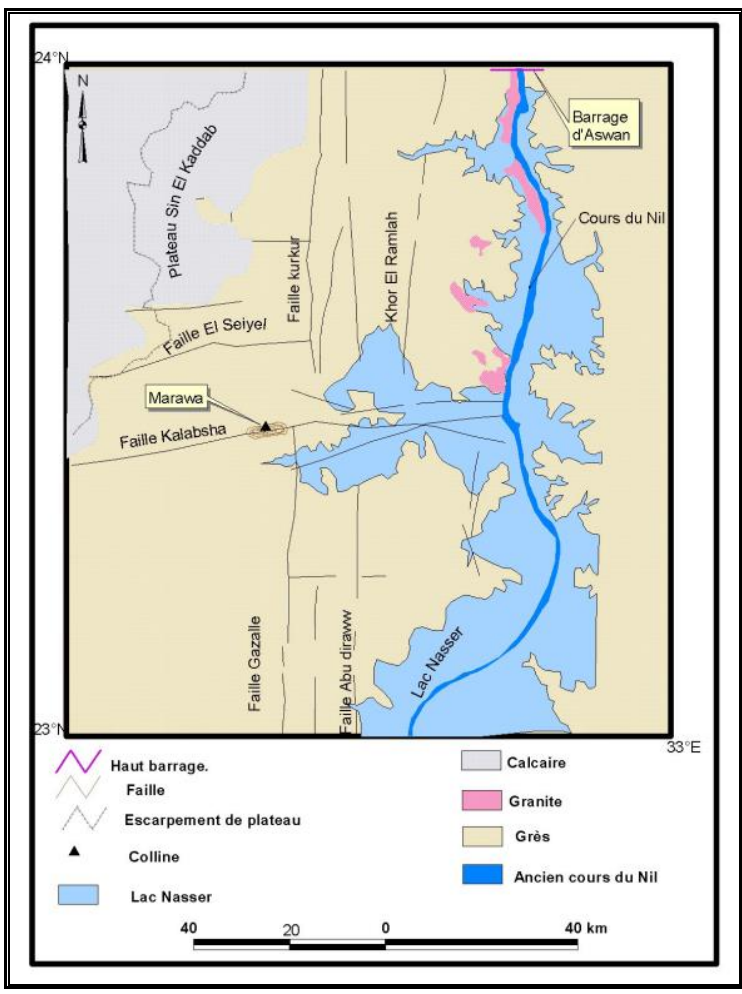

Figure 3. Carte géologique de la partie occidentale du réservoir du lac Nasser

(Zone Kalabsha). (d'après la carte géologique 1/ 50 000, 1987)

Le service géologique Egyptien (1981) a confirmé que la faille de Kalabsha approximativement 300 kilomètres de long. La faille est presque remplie de ciment siliceux ou ciment d'oxyde de fer et ce ciment est plus résistant à l'érosion que le grès de Nubie.

\section{2-2-3. Le niveau d'eau et l'inondation du Lac} Nasser

Afin d'expliquer tout rapprochement entre le niveau du réservoir et la séismicité à Assouan, il est nécessaire de comprendre la relation entre le niveau d'eau et l'ampleur latérale du réservoir. Le réservoir d'Assouan est situé sur un terrain relativement plat. Parfois, pendant les périodes de sécheresse, la quantité des eaux dans le réservoir se limite pendant l'étiage du Nil.

Le tableau ci-dessous (2-2) montre les changements dans les niveaux d'eau du Lac à partir de 1966 jusqu'à 1998 
Tableau 1. Les changements dans les niveaux d'eau dans le Lac pour la période 1966-1998. (d'après l'autorité du lac Nasser ,2000)

\begin{tabular}{|l|l|l|l|l|l|l|l|}
\hline Année & $\begin{array}{c}\text { Hauteur } \\
\text { d'eau }(\mathrm{m})\end{array}$ & Année & $\begin{array}{c}\text { Hauteur } \\
\text { d'eau }(\mathrm{m})\end{array}$ & Année & $\begin{array}{c}\text { Hauteur } \\
\text { d'eau }(\mathrm{m})\end{array}$ & Année & $\begin{array}{c}\text { Hauteur } \\
\text { d'eau }(\mathrm{m})\end{array}$ \\
\hline 1966 & 141 & 1974 & 171 & 1982 & 172 & 1990 & 168 \\
\hline 1967 & 151 & 1975 & 176 & 1983 & 169 & 1991 & 169 \\
\hline 1968 & 156 & 1976 & 177 & 1984 & 166 & 1992 & 170 \\
\hline 1969 & 161 & 1977 & 177 & 1985 & 164 & 1993 & 174 \\
\hline 1970 & 164 & 1978 & 178 & 1986 & 162 & 1994 & 177 \\
\hline 1971 & 167 & 1979 & 177 & 1987 & 158 & 1995 & 176 \\
\hline 1972 & 165 & 1980 & 176 & 1988 & 168 & 1996 & 178 \\
\hline 1973 & 166 & 1981 & 176 & 1989 & 169 & 1997 & 178 \\
\hline & \multicolumn{7}{|r|}{} \\
\hline
\end{tabular}

Les faibles changements de la profondeur de l'eau dans le réservoir amènent de grands changements de la superficie du lac. La séismicité à Assouan a été confinée principalement dans la zone d'oued Kalabsha où elle situe à l'ouest du réservoir (fig. 2-5). Cette zone a commencé à se remplir après que le niveau d'eau dans le réservoir ait excédé $165 \mathrm{~m}$ en 1974.

De 1975 à 1985, l'ampleur régionale du réservoir dans la région de Kalabsha a changé sensiblement. Les changements les plus importants ont eu lieu lors de l'inondation de 1981 et au moment des vidanges successives pendant les périodes de sécheresse dans la dépression d'Abou Riheiwa au Nord du Jbel Marawa (fig 4). La diminution du niveau d'eau dans la dépression a été caractérisée par la formation de plusieurs chenaux séparés par des affleurements rocheux.

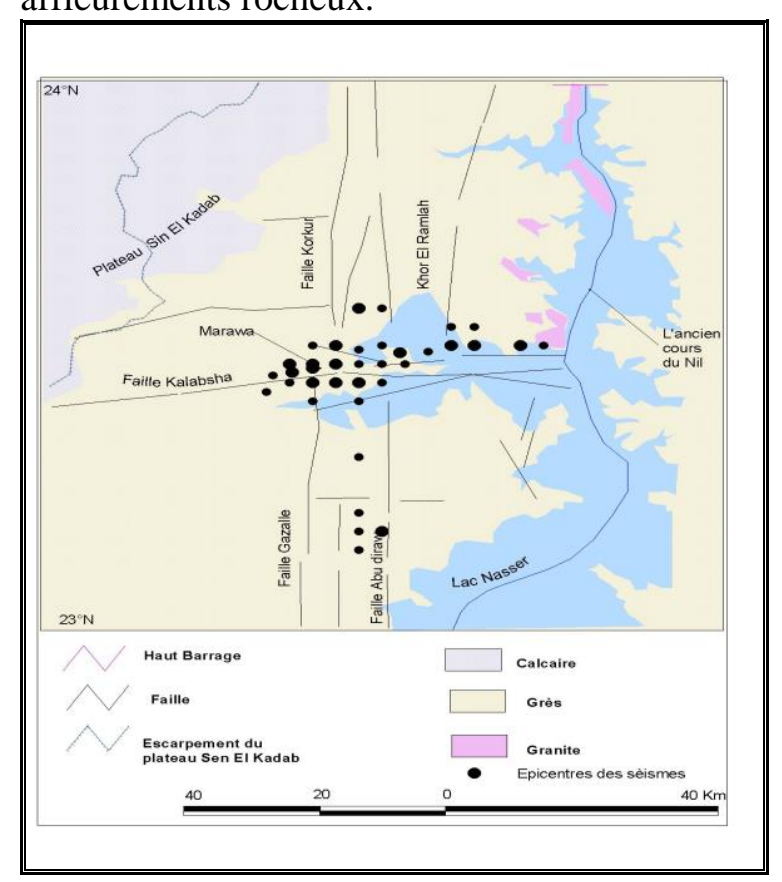

Figure 4. La partie nord du Lac Nasser et la séismicité d'Assouan enregistrées par le réseau télémétré. (Zone de Kalabsha) (d'après Simpson, Gharib et Kebeasy, 1989)
2-3. Les changements du niveau de l'eau dans le lac et la séismicité

Les variations temporelles de la sismicité à Assouan et les changements du niveau de l'eau dans le réservoir sont montrées dans la figure 5 . Le tremblement de terre du 14 novembre 1981 s'est produit immédiatement après que le niveau d'eau saisonnier maximum ait été atteint. Les activités séismiques étaient également évidentes en août 1982, décembre janvier 1983 et juin 1987. Il y a eu une diminution générale du taux d'activité séismique depuis le tremblement de terre de novembre 1981 particulièrement lors du séisme du 14 novembre sous du Jbel Marawa (fig. 5).

La figure (5) montre la variabilité temporelle dans le niveau du réservoir et ses fluctuations journalières. Les changements du niveau de la sismicité sont détectés par le réseau pour chaque jour et pendant une période de dix jours (la période est couverte par l'opération du réseau télémétré d'après Simpson, Gharib, et Kebeasy (1989) les plus grands tremblements de terre sont indiqués dans la figure (6). Dans la section suivante et la figure (6) nous présentons un bref résumé sur la relation entre les changements du niveau de l'eau dans le lac Nasser et la sismicité :

\section{- Novembre 1981 :}

La secousse sismique a commencé le 14 novembre 1981, quatre jours après le remplissage complet du réservoir du 10 novembre 1981. La profondeur de l'événement ne peut pas être évaluée parce qu'il n'avait aucun instrument local fonctionnant au début du séisme.

-Août - Septembre 1982 :

Le plus grand tremblement de terre qui s'est produit depuis le tremblement de terre de novembre - décembre 1981; était de 4,69 sur l'échelle de Richter et s'est produit le 20 août 1982 sous le Jbel Marawa, dans le même secteur où le tremblement de novembre 1981 s'est déclenché. Il a été suivi de secousses séismiques pendant 9 jours, et le niveau de l'eau est resté élevé jusqu'au début de septembre. Le minimum saisonnier du niveau d'eau a été atteint le 11 août 1982, 9 jours avant le tremblement de terre du 20 Août. 


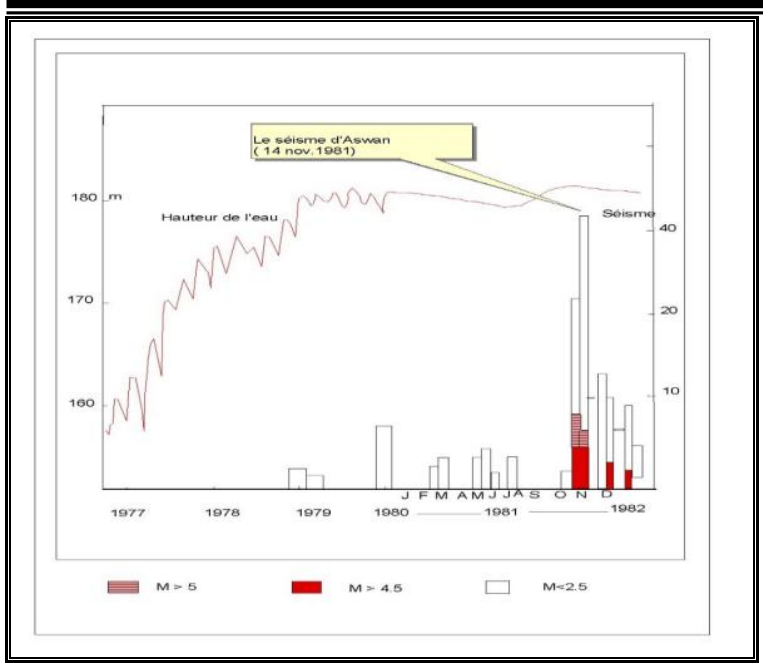

Figure 5. Corrélation entre le niveau d'eau dans le réservoir d'Assouan et les séismes avant et après le grand séisme d'Assouan du 14 novembre 1981.

(d'après Simpson et al. 1989)

\section{-Novembre 1982 - Mars 1983}

Les problèmes d'enregistrement de bandes du réseau à la fin de l'été de 1982 rendus difficiles la localisation véritable des secousses séismiques pendant le mois de septembre et de Novembre. Les enregistrements sur les séismogrammes ont visiblement continué. Le niveau de l'eau a atteint son maximum le 13 novembre, 9 jours avant le commencement de la séismicité. Un tremblement de terre de 4,2 sur l'échelle de Richter s'est produit à la fin de cette période (24 février 1983).

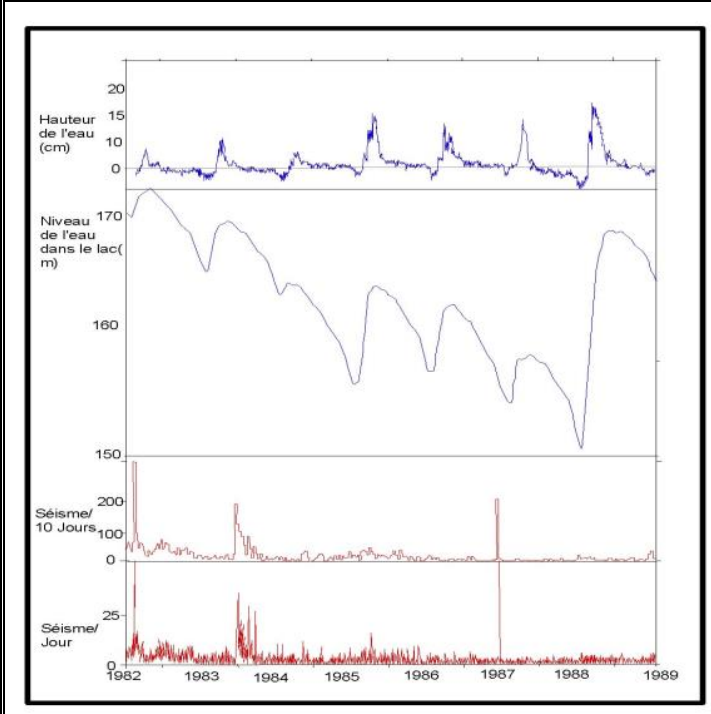

Figure 6. La variation temporelle dans le niveau du réservoir et ses fluctuations journalières et les nombres d'événements détectés par le réseau pendant dix jours et pendant un jour. (D'après Simpson, Ghrib, et Kebeasy 1989)

\section{-Décembre 1983 - Mars 1984}

L'augmentation des activités sismiques a commencé à la fin de 1983 à des profondeurs faibles au Nord-Est du Jbel Marawa. A ce moment, le niveau du lac a baissé suffisamment pour entraîner la vidange de la dépression d'Abou Riheiwa et sur tout le secteur au Sud de Marawa

A la fin de cette période, deux tremblements de terre de 3,4 sur l'échelle de Richter se sont produits pendant la crête mineure de la crue de février. Le niveau d'eau a commencé à baisser le 10 décembre, et la séismicité a commencé le 22 décembre, 12 jours plus tard.

\section{-Novembre - décembre 1984}

Le changement annuel du niveau de l'eau en 1984 est nettement moins important que les années précédentes, et le niveau maximum de l'eau n'a jamais était aussi bas depuis 1975. Le niveau de l'eau a commencé à diminuer le 20 Octobre, et une augmentation mineure de la séismicité a commencé le 13 novembre. Les hypocentres pour ces événements sont exactement situés au Nord-Est du Jbel Marawa.

\section{-Juin 1987}

Un tremblement de terre peu profond au Nordest de Marawa a commencé le 15 juin 1987 de 3,7 sur l'échelle de Richter dix jours plus tôt, le 5 juin, il y avait eu une augmentation du taux d'abaissement du niveau de l'eau dans le lac, de 3 centimètres par jour en moyenne, jusqu'au premier Juin, 8 centimètres par jour, juste avant le commencement des activités le 15 Juin. Le niveau de l'eau dans cette période était le niveau le plus bas depuis 16 ans. Il n'y a aucun changement évident des niveaux des activités sismiques avec les changements du niveau de l'eau pendant l'année.

$-1988$

L'activité a continué de manière faible avec la diminution du niveau de l'eau de 1985 à 1988, il n'y a aucune augmentation évidente de la séismicité liée aux maximums saisonniers du niveau de l'eau dans cette période. En 1985 et 1988 , il y a une grande augmentation d'activité pendant le milieu de l'année, près de la période des minimums de niveau de l'eau. Le niveau de l'activité est demeuré relativement constant tout au long de 1986

\section{-Juin - Juillet 1989}

L'augmentation au niveau de l'activité sismique en juillet 1989 est intéressante en raison de sa similitude avec l'activité sismique 
en juin 1987. L'augmentation des activités sismiques en 1989 se produit sur approximativement le même laps de temps. A la fin de l'année 1989, les importantes activités séismiques se sont arrêtées grâce au remplissage complet du lac qui a exercé une certaine pression sue la zone et par conséquence a limité le libre mouvement des couches rocheuses de ce dernier, favorisant une quasi stabilité voire une stabilité complète témoignée par le manque des activités sismiques dans la région depuis 1989.

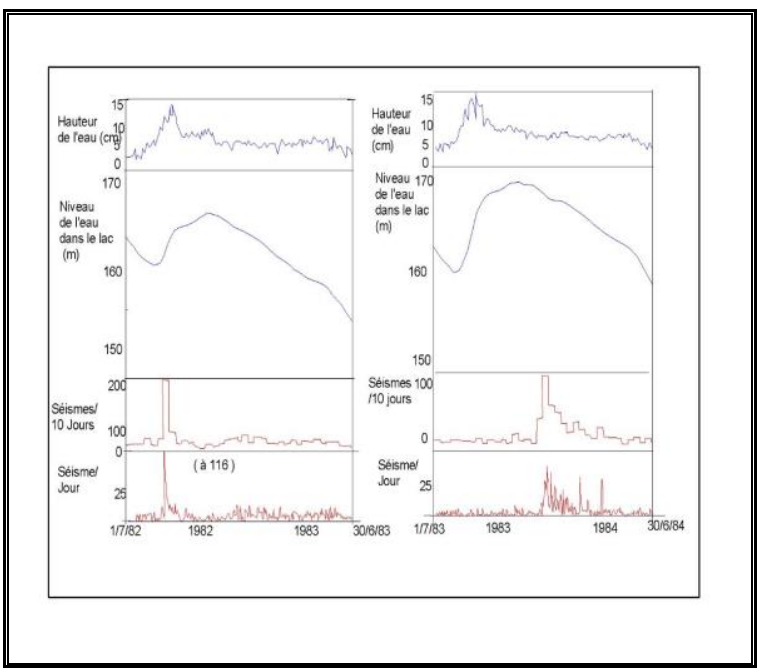

Fig-7. -Deux graphiques des séismicités en août 1982 et décembre 1983- février 1984(d'après Simpson et al. 1989)

\section{Distributions des hypocentres et des épicentres}

Les tremblements de terre de Kalabsha (fig.7), de 1982 à 1990, se sont produits dans deux zones d'hypocentre, peu profondes, séparées par les zones de Marrwa

1-Zone peu profonde qui est définie par une profondeur maximale d'environ 12 kilomètres, et les épicentres de ces tremblements de terre ont été partagés en quatre secteurs :

a- Au Nord de la faille de Kalabsha (Khor El

Ramla et faille du Sayal) lorsque le niveau de l'eau a dépassé $165 \mathrm{~m}$.

b- Au sud de la faille de Kalabsha, et Nord de la faille Gazelle et faille d'Abou Diraw.

c- À l'Est du chenal principal du Nil.

d- L'ensemble de deux zones d'activité sismiques, l'une située le long de la faille de Kalabsha et l'autre située le long de la partie orientale du Jbel Marawa, à proximité du chenal principal du Nil.

2- Une zone plus profonde, où la profondeur locale est plus de $12 \mathrm{~km}$. Cette zone est toujours en activité et les épicentres sont sous la colline du Jbel Marawa (Kebesy, 1990).

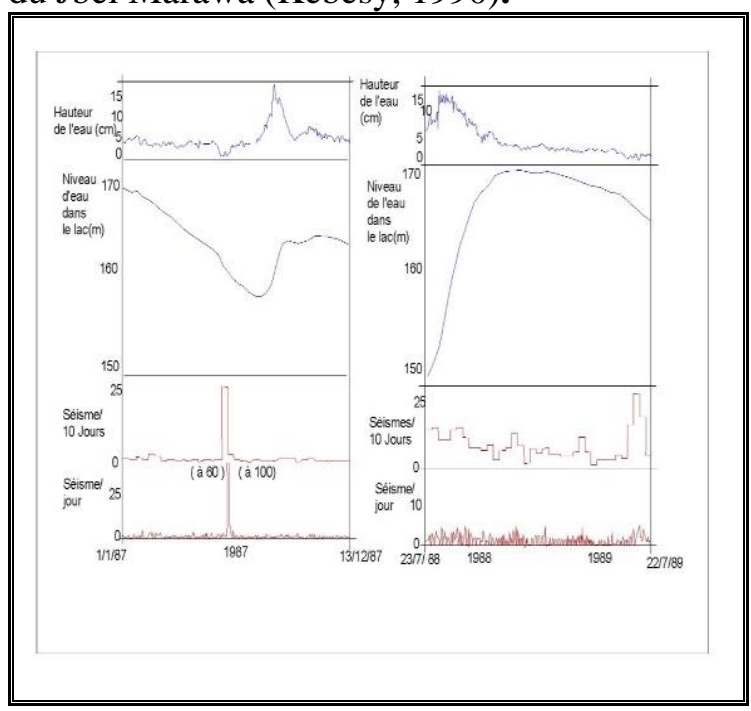

Figure-8. Deux graphiques des séismicités en juin 1987 et juillet 1989

(d'après Simpson et al. 1989)

\section{5-1. Les effets de site.}

On appelle effets de site les effets d'amplification dus aux caractéristiques géométriques du sol. La géomorphologie d'Assouan est composée de formations souvent très contrastées par leurs propriétés mécaniques avec des géométries complexes (topographie, faille..).

Aussi les ondes sismiques peuvent -elles subir de multitudes réflexions, réfractions, diffractions, conduisant à des mouvements observés en surfaces très variables suivant le site (fig.8). La configuration très répandue (bassins sédimentaires, vallées alluviales, etc....) constitue un "piège » pour les ondes sismiques, et il s'ensuit, à certaines fréquences, des amplifications dépendant $\mathrm{du}$ contraste mécanique entre surface et profondeur

D'après l'échelle Richter des séismes (tableau 2) nous avons classé les effets de séisme en cinq parties comme suit :

Tableau 2. Les magnitudes de séismes et dégâts (d'après d'échelle Richter)

\begin{tabular}{|l|l|}
\hline Magnitude & Effet de séisme \\
\hline$<2,5$ & $\begin{array}{l}\text { En général, très faible et sans } \\
\text { conséquences }\end{array}$ \\
\hline 4,5 & Dégâts locaux. \\
\hline 6 & Dégâts un peu intenses \\
\hline 7 & Beaucoup de dégâts \\
\hline 8 & Grands dégâts \\
\hline
\end{tabular}




\section{5-1-1. L'écroulement :}

Ce type de processus se trouve à $50 \mathrm{~km}$ au Sud-est d'Assouan près de la fontaine Omhebal. La photo (1) ci-dessous montre l'écroulement des blocs de granosyénite lors du tremblement de terre. Le poids de ces blocs varie d'un kilogramme à 20 tonnes (EL Shazely, 1982), les traces d'arrachements bien visibles, montrent que le séisme était le facteur principal de ces écroulements.

\section{5-1-2. Les fissurations}

La zone fissurée est située à $55 \mathrm{~km}$ au sud-ouest d'Assouan dans le désert libyque. La photo (2) ci-dessous montre les diaclases dans le grès clair en surface d'orientation est sud-est-, ouest -nord-ouest.

D'après ELSHAZLAY Les formes, les fissures et les diaclases qu'on voie sur les photos sont liées essentiellement au séisme du 14 novembre 1981

Généralement ces fissures ont les directions suivantes :

Nord - Nord-Est -------- Sud/Sud- Ouest

Nord-Est----------------Sud-Ouest

Est / Sud-Est--------------Ouest /Nord-Ouest

Nord-Ouest-----------------Sud-Est

Nord /Nord-ouest---------Sud,/Sud-Est

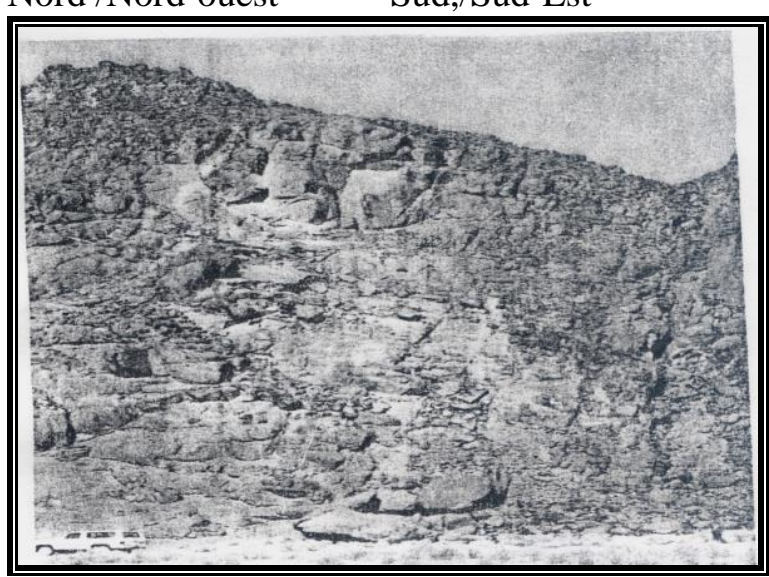

Photo 1. Ecroulements des blocs $\mathrm{de}$ Granosyénites pendant le séisme du 14 nov.1981 (50 km au Sud de la ville d'Assouan, d'après El Shazly)

Parfois une fissure prend trois directions, le Nord-Est, le Sud/Sud-Est, le Nord/ Nord-Est, le Sud Sud-ouest, et le Nord Nord-ouest et Sud Sud-est

Ces fissures ont des longueurs de $50 \mathrm{~cm}$ à $1 \mathrm{~km}$, et au moins de $1 \mathrm{~mm}$ à $1 \mathrm{~m}$ de largeur. On a retrouvé des pierres tombées sur les plans des failles de Kalabsha (photo 2).

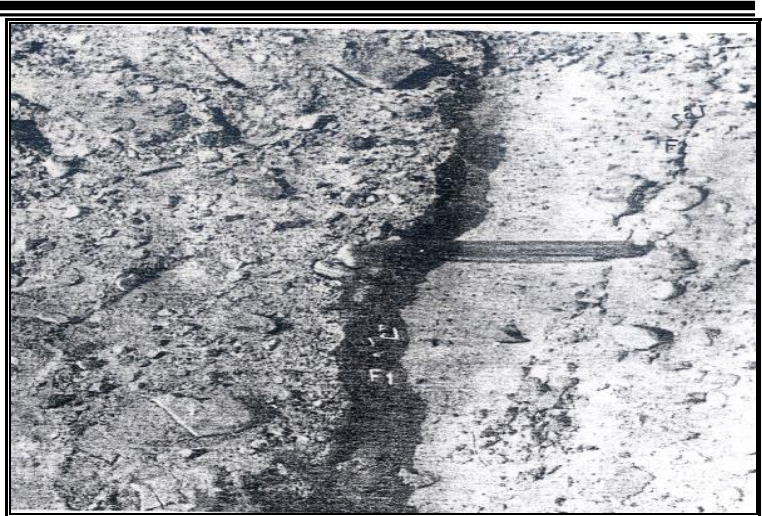

Photo 2- Une faille de direction Est/ Nord-Est et Ouest/ Sud-Ouest.

(Oued Kalabsha au $80 \mathrm{Km}$ au sud-ouest d'Assouan d'après El Shazly)

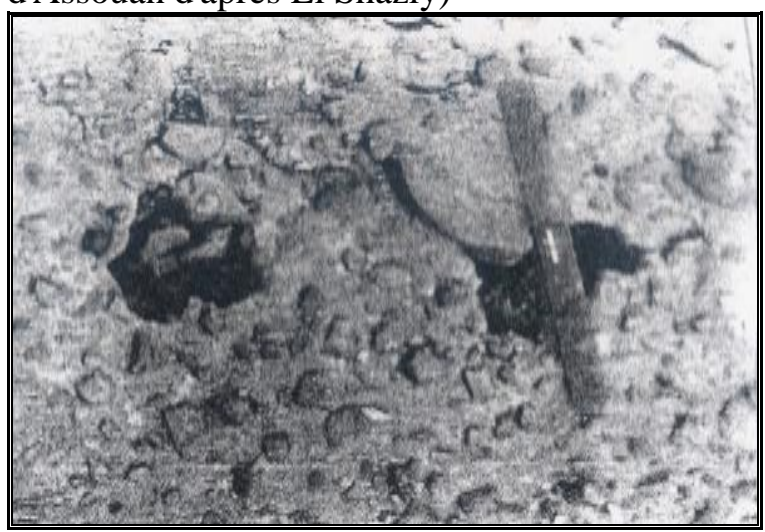

Photo 3.Une faille de direction Est Sud-Est Ouest Nord-Ouest

dans le grès,

$(80 \mathrm{~km}$ au Sud-Ouest de la ville d'Assouan d'après ELShazly)

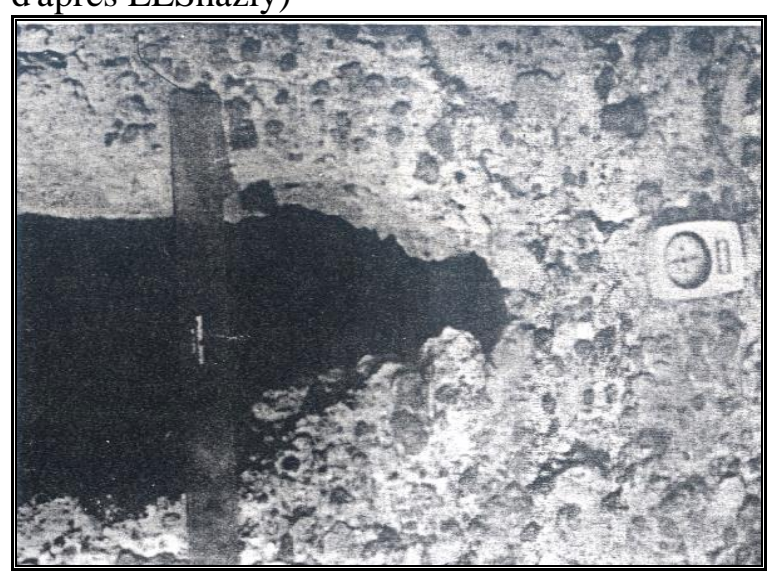

Photo 4. Rejeu d'une faille de direction Nord /Nord-Est -et Sud /Sud-Ouest coupant la formation de grès marneux en surface

$(80 \mathrm{~km} \mathrm{au}$ Sud-Ouest de la ville d'Assouan d'après El Shazly)

\section{La prévision}

Pour ces risques, il consisterait à définir, grâce à un certain nombre de méthodes : la date, le lieu et l'intensité du phénomène afin que 
toutes les mesures puissent être prises de façon efficace.

Pour les séismes, la prévision temporelle est difficile ainsi que la prévision de l'intensité qui est primordiale. La prévision spatiale semble évidente, s'il se produit un séisme, il touchera toute la ville d'Assouan et ses villages mais, des nuances seront apportées du fait que l'impact ne sera pas uniforme, ceci sera établi sur une cartographie.

Nous appliquons ici une méthode probabiliste qui devrait être complétée par un méthode déterministe. La première consiste donc à identifier les zones à risques en tenant compte de la nature des sols et de la tectonique générale mais aussi en recensant les catastrophes historiques. La seconde étudie les signes précurseurs et nécessite des instruments de surveillance continue

\section{6-1. Y a-t-il une récurrence dans l'apparition} des phénomènes sismiques ? Peut -on prévoir leurs intensités.

Il s'agit en effet d'effectuer une étude statistique en déterminant tous les séismes susceptibles d'avoir touché Assouan. Pour ce faire, nous avons utilisé la source de l'Institut des séismes au Caire. Tout ceci dans le but de savoir s'il y a une récurrence dans l'apparition des séismes pour déterminer dans combien de temps aura lieu le prochain et quelle intensité il pourra avoir.

Assouan n'est pas clairement cité dans beaucoup de séismes, et nous avons donc été contraints d'estimer si la ville avait ou non été affectée, en essayant de rester le plus objectif possible. Nous avons donc évidemment considéré les événements dans lesquels Assouan était explicitement cité, mais aussi ceux ayant affecté Abou Semble, Gharb Assouan, voire plus largement dans le Sud d'Egypte. Cette étude nous a permis de créer le graphique (9) qui montre le nombre de séismes qui auraient affecté Assouan de 1981 à 1992.

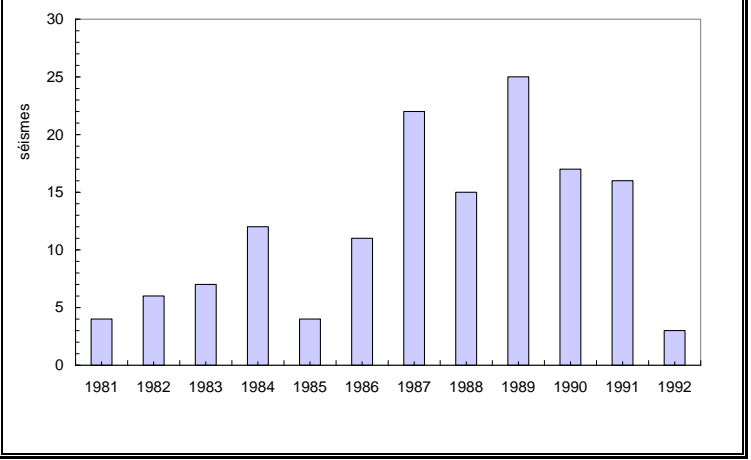

Figure 9. Nombres de séismes à Assouan entre 1981 et 1992.

(d'après Institut Helwan des séismes, 1992)

L'étude du graphique révèle qu'il y a eu 132 séismes en 12 ans, ce qui fait une moyenne d'environ onze séismes chaque année. Le graphique aussi montre que certaines années sont l'objet de 25 séismes en (1987), alors que certaines années n'en comportent pas beaucoup 3 en 1992. Ces opérations statistiques confirment mathématiquement l'irrégularité des séismes.

En conclusion, nous pouvons dire que les phénomènes séismiques sont récurrents mais non réguliers. Il est par conséquent impossible de prévoir quand va survenir un séisme via une étude statistique. De même, l'intensité des futurs séismes n'est pas déterminable. La seule certitude est que la zone concernée est toujours active et l'on peut être certain que d'autres séismes surviendront dans l'avenir.

\section{6-1-1. Contre les séismes.}

A Assouan, aucune de ces précautions n'a pas été prise. En effet, il y a de nombreuses constructions sur les versants escarpés à El Mahmodia (fig. 10), sur des terrains meubles (les argilites), à proximité d'une faille active (celle de Toushka) même si celle-ci n'est pas visible en surface, et aux bords des bancs de grés.

On se rend compte que sur le même espace, le bilan serait très lourd en pertes humaines. Pour situer Assouan dans cet espace, il faut dire qu'Assouan avait moins de 5000 habitants, en 1981. Aujourd'hui, elle approche des 10000 habitants. En 1981, il n'y avait pas eu de morts mais aujourd'hui, avec l'augmentation de population le bilan devrait être lourd si un séisme comme celui de 1981 touchait de nouveau Assouan. 
Du fait du manque de prévention, si un séisme comme celui de 1981 se produisait, il faudrait s'attendre à un scénario catastrophique avec des pertes humaines et économiques considérables. Afin d'éviter un tel désastre, la meilleure solution serait peut-être d'anticiper ceci et d'établir des normes de constructions parasismiques.

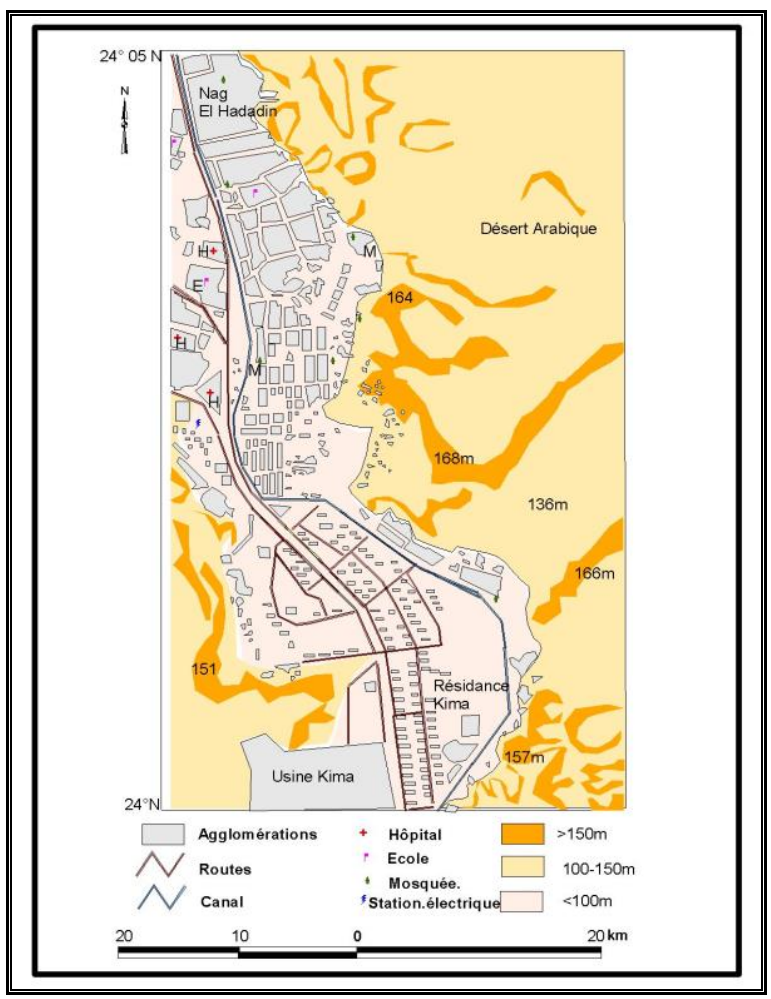

Figure 10. Carte de localisation à El Mahmodia à Assouan

(d'après la carte topographique $1 / 25000$ )

\section{7- Les résultats}

-Les conditions géologiques, géomorphologiques et sismologiques sur le secteur occidental du réservoir du Haut Barrage ont été étudiées dans le but de déchiffrer la nature de l'interdépendance entre la fluctuation du niveau d'eau du réservoir et l'activité sismique depuis 1964 où le réservoir a commencé à se remplir .L'analyse de cette interdépendance entre le niveau d'eau et la séismicité dans le secteur selon les données du réseau de station de Nice, il n'y a aucun enregistrement, sur n'importe quel disque, d'activité sismique avant la construction du Haut Barrage d'Assouan. Mais après que le réservoir ait commencé à se remplir, un tremblement de terre de 5,3 sur l'échelle de Richter s'est produit le 14 novembre 1981 dans la région d'Assouan, suivi de séries de répliques sismiques qui se sont produites dans la région le long de la faille de Kalabsha.

-Du niveau de $175 \mathrm{~m}$ d'eau dans le réservoir, le secteur du Jbel Marawa situé le long de la faille du Kalabsha, est inondé et les épicentres des tremblements de terre dans cette période d'inondation se caractérisent par des foyers plus profonds que 12 kilomètres. Tous les tremblements de terre de 3,5 ont des hypocentres sous Jbel Marawa. Quand le niveau de l'eau est plus ou moins de $165 \mathrm{~m}$, l'eau se trouve aux limites du cours du Nil (le cours du Nil avant la construction du Haut Barrage) et les épicentres des tremblements de terre dans cette période sont profonds de $12 \mathrm{~km}$. L'activité sismique augmente toujours dans la période décroissant du niveau d'eau du réservoir

-Les tremblements de terres égales ou supérieures de 3,5 sur l'échelle de Richter sont toujours produits quand le niveau de l'eau du réservoir est plus élevé que $165 \mathrm{~m}$, où le Jbel Marawa le long de la faille de Kalabsha est inondé.

- Les dix-sept ans entre le début du remplissage du réservoir en 1964 et le commencement de l'activité sismique en novembre 1981 étaient liés à l'inondation de la région de Marawa qui a commencé en1975, quand le niveau de l'eau a atteint $175 \mathrm{~m}$.

\section{Conclusion}

Le tremblement de terre du 14 novembre 1981 est le plus grand événement séismique produit dans la région d'Assouan dans l'histoire. Les données instrumentales limitées suggèrent que les tremblements de terre de petite intensité ont pu se produire dans le secteur pendant les 5 années précédentes. Mais les épicentres de tels événements sont inconnus. Ainsi, le tremblement de terre 1981 et la séismicité associée représentent une augmentation soudaine au niveau de la séismicité régionale.

Le tremblement de terre de novembre 1981 a été concentré le long d'une partie de 15 kilomètres de l'extrémité orientale de la faille de Kalabsha aux profondeurs de 15 à 20 kilomètres. On observe plusieurs subzones d'une activité moins profonde de tremblement de terre (des profondeurs de 0 à 10 kilomètres) à l'Est de l'épicentre de 1981, le long de la faille de Kalabsha. .

Le tremblement de terre de novembre 1981 s'est produit juste après le maximum saisonnier du niveau d'eau dans le réservoir. On 
a observé de tremblements de terre au niveau de l'activité de séismes peu de temps après le maximum saisonnier du niveau d'eau en 1981, 1982, 1983 et 1984.

Les pics dans la séismicité profonde n'ont pas continué, le niveau du lac est tombé au-dessous des requis pour inonder la région de Kalabsha. Les pics de la séismicité moins profonde n'ont commencé à être enregistrés qu'à partir de ces deux dernières années qui ont suivi le tremblement de terre de 1981. Le tremblement de terre du 20 Août1982 était la plus grande magnitude 4,6 sur l'échelle Richter juste après le minimum saisonnier du niveau d'eau.

Généralement, les dégâts dans les constructions ont été faibles en 1981. Il n'y avait pas d'importantes constructions en béton à Assouan. La plupart des constructions, sont faites avec des limons et des matériaux traditionnels, constitué d'un seul ou deux étages maximum. Actuellement, si un tremblement de terre de 5,3 sur l'échelle de Richter affecte Assouan, les dégâts seront plus importants que les précédents à cause de la croissance de la population et des constructions.
References:

- DESSOKEY M., KEBEASY M. \& HASEAB H. (1985): Preliminary estimation of seismic hazard in the Aswan Lake Area in Egypt., ACTA GEOPHYSICA POLONICA Vol. XXXIII, no.3.

- EL SHAZLY E. (1982): the effete of the earthquake in the Aswan area. Geological Survey of Egypt. Government Press, pp 1572

- HEMDAN G. (1984): Characterize of Egypt, (en Arabe) Part 1 Dar El HELAL, Cairo, 841p

- ISSAWI B. (1978) : Geology of Nubian west area, western Desert- Egypt, Ann.Geol.Survey Egypt, 3, pp 237-253.

- KEBEASY R. MAAMOUN M. \& IBRAHIM E. (1982): Aswan lake earthquakes, Bull. Inter. Inst. Seism. Earthq. Engin. Tokyo, 19, pp155-160.

- KIJKO A. DOSSOKY, M. KEBESY R. \& HASEAB G. (1985): Preliminary estimation of seismic hazard in the Aswan Lake area in Egypt. Acta Geophysica Polonica, vol.XXXIII, $n^{\circ} 3$, pp269-277.

- SIMPSON D. GHARIB A. \& KEBEASY, R. (1989) : Induced seismicity and changes in water level at Aswan reservoir, Egypt. Gerlands Beitr zur Geophysik, Vol.99 n³, pp191-204.

- WOODWARD \& Clyde Consultants. (1985): Seismicity studies of the Aswan région: Earthquake Activity and Dam stability Evaluations for the Aswan High Dam, Ministry of Irrigation, Arab Republic of Egypt, Walnut Creek, CA.123 p 